N. Yamauchi

Nagoya Math. J.

Vol. 83 (1981), 107-121

\title{
ON ALGEBRA WITH UNIVERSAL FINITE MODULE OF DIFFERENTIALS
}

\author{
NORIO YAMAUCHI
}

Let $k$ be a field and $A$ a noetherian $k$-algebra. In this note, we shall study the universal finite module of differentials of $A$ over $k$, which is denoted by $D_{k}(A)$. When the characteristic of $k$ is zero, detailed results have been obtained by Scheja and Storch [8]. So we shall treat the positive characteristic case. In $\S 1$, we shall study differential modules of a local ring over subfields. We obtain a criterion of regularity (Theorem (1.14)). In $\S 2$, we shall study the formal fibres and regular locus of $A$ with $D_{k}(A)$. Our main result is Theorem (2.1) which shows that, if $D_{k}(A)$ exists, then $A$ is a universally catenary $G$-ring under a certain assumption. In the local case, this is a generalization of Matsumura's theorem ([5] Theorem 15), where regularity of $A$ is assumed.

Throughout this note, rings are commutative with unit element. We freely use the notation and the terminology in [2].

\section{§0. Preliminaries}

First we summarize generalities of universal finite module of differentials. For the detail, see [8].

Let $A$ be a ring and $M$ an $A$-module. We say that $M$ is a prefinite $A$-module if the following condition is satisfied:

For any non-zero element $m$ of $M$, there is a finite $A$-module $N$ and an $A$-linear map $f: M \rightarrow N$ such that $f(m) \neq 0$.

$M$ is a prefinite $A$-module if and only if it is a submodule of a direct product of finite $A$-modules.

For an $A$-module $M$, we put $K=\{m \in M \mid$ for any finite $A$-module $N$ and any $A$-linear map $f: M \rightarrow N, f(m)=0\}$ and $\bar{M}=M / K$. Let $p: M \rightarrow \bar{M}$ be the natural surjection.

Received June 29, 1979.

Revised October 30, 1979. 
Proposition (0.1). Let the notation be as above. Then:

(1) $\bar{M}$ is a prefinite A-module.

(2) If $N$ is a prefinite A-module, then there is a natural isomorphism of A-modules: $\operatorname{Hom}_{A}(M, N) \simeq \operatorname{Hom}_{A}(\bar{M}, N)$.

(3) If $f: M \rightarrow N$ is a homomorphism of A-modules, then there is a unique A-linear map $\bar{f}: \bar{M} \rightarrow \bar{N}$ such that $p^{\prime} \circ f=\bar{f} \circ p$ where $p^{\prime}$ is the natural surjection $N \rightarrow \bar{N}$. Thus $M \mapsto \bar{M}$ is a covariant functor from the category of A-modules into the category of prefinite A-modules.

Proof. Let $N$ be an arbitrary prefinite $A$-module and let $f: M \rightarrow N$ be an $A$-homomorphism. Then it is easy to see that $\operatorname{ker}(f) \supset K$. The assertions follow from this fact.

Let $k$ be a ring and $A$ be a $k$-algebra. Let $D$ be an $A$-module and $d: A \rightarrow D$ a $k$-derivation. $D$ is called the universal prefinite (resp. finite) module of differentials of $A$ over $k$ if the following conditions are satisfied (cf. [8]):

(1) $D$ is a prefinite (resp. finite) $A$-module and $D=A d A$,

(2) for any prefinite (resp. finite) $A$-module $M$ and $k$-derivation $\delta: A$ $\rightarrow M$ there is a unique $A$-linear map $f: D \rightarrow M$ such that $\delta=f \circ d$. (i.e. $\operatorname{Der}_{k}(A, M) \simeq \operatorname{Hom}_{A}(D, M)$.)

Proposition (0.2). Let $k$ be $a$ ring and $A$ a k-algebra. Then:

(1) The universal prefinite module of differentials of $A$ over $k$ exists and is unique up to isomorphism.

(2) The universal finite module of differentials of $A$ over $k$ exists if and only if the universal prefinite module of differentials of $A$ over $k$ is $a$ finite $A$-module.

Proof. (1) Let $\Omega_{A / k}$ be the usual module of differentials and put $D=\overline{\Omega_{A / k}}$. Then $D$ is the universal prefinite module of differentials by (0.1).

(2) The "if" part is obvious by the definition, while the "only if" part is easy since any prefinite module can be embedded into a direct product of finite modules. (cf. [8] (1.1).)

We denote by $D_{k}(A)$ the universal finite module of differentials of $A$ over $k$. The canonical derivation $d: A \rightarrow D_{k}(A)$ is called the universal finite $k$-derivation of $A$.

Proposition (0.3). Let $k$ be $a$ ring and $A$ a noetherian $k$-algebra with $D_{k}(A)$. Then: 
(1) If $\mathfrak{m}$ is a maximal ideal of $A, D_{k}\left(A_{\mathfrak{m}}\right)$ exists and $D_{k}\left(A_{\mathfrak{m}}\right) \simeq D_{k}(A)_{\mathfrak{m}}$

(2) If $A$ is a local ring, $D_{k}(\hat{A})$ exists and $D_{k}(\hat{A}) \simeq D_{k}(A) \otimes_{A} \hat{A}$ where $\hat{A}$ denotes the completion of $A$.

Proof. See [8] (1.8) and (1.6).

Proposition (0.4). Let $k, A$ and $B$ be noetherian rings and $k \stackrel{f}{\longrightarrow} A$ $\stackrel{g}{\longrightarrow} B$ be homomorphisms of rings. Then:

(1) If $D_{k}(B)$ exists, so does $D_{A}(B)$.

(2) Assume that any prefinite B-module is also a prefinite A-module. Then, when $D_{k}(A)$ and $D_{k}(B)$ exist, there is an exact sequence of natural homomorphisms of B-modules:

$$
D_{k}(A) \otimes_{A} B \rightarrow D_{k}(B) \rightarrow D_{A}(B) \rightarrow 0 .
$$

(3) If $D_{k}(A)$ exists and $g$ is surjective, then $D_{k}(B)$ exists and we have the following exact sequence of $B$-modules where $I=\operatorname{ker}(g)$ :

$$
I / I^{2} \rightarrow D_{k}(A) \otimes_{A} B \rightarrow D_{k}(B) \rightarrow 0 .
$$

Proof. (1) Trivial by (0.2).

(2) Let $M$ be an arbitrary finite $B$-module. Then we have the following exact sequence of natual homomorphisms:

$$
0 \rightarrow \operatorname{Der}_{A}(B, M) \rightarrow \operatorname{Der}_{k}(B, M) \rightarrow \operatorname{Der}_{k}(A, M) .
$$

By definition,

$\operatorname{Der}_{A}(B, M) \simeq \operatorname{Hom}_{B}\left(D_{A}(B), M\right)$ and $\operatorname{Der}_{k}(B, M) \simeq \operatorname{Hom}_{B}\left(D_{k}(B), M\right)$.

Since $M$ is a prefinite $A$-module by the assumption, $\operatorname{Der}_{k}(A, M) \simeq$ $\operatorname{Hom}_{A}\left(D_{k}(A), M\right) \simeq \operatorname{Hom}_{B}\left(D_{k}(A) \otimes_{A} B, M\right)$. Therefore the sequence

$$
0 \rightarrow \operatorname{Hom}_{B}\left(D_{A}(B), M\right) \rightarrow \operatorname{Hom}_{B}\left(D_{k}(B), M\right) \rightarrow \operatorname{Hom}_{B}\left(D_{k}(A) \otimes_{A} B, M\right)
$$

is exact. Thus $(*)$ is exact.

(3) The existence of $D_{k}(B)$ is obvious since $\Omega_{B / k}$ is a homomorphic image of $\Omega_{A / k}$. The exactness of $(* *)$ follows from the fact that for any finite $B$-module $M$, the following sequence is exact:

$$
0 \rightarrow \operatorname{Hom}_{B}\left(D_{k}(B), M\right) \rightarrow \operatorname{Hom}_{B}\left(D_{k}(A) \otimes_{A} B, M\right) \rightarrow \operatorname{Hom}_{B}\left(\left(I / I^{2}, M\right) .\right.
$$

Remark. The assumption of (2) is satisfied in the following cases: 
(a) $A$ is a field. (b) $g$ is a local homomorphism of noetherian local rings. (cf. [8] (1.3)).

Proposition (0.5). Let $k$ be $a$ ring and $A$ a noetherian $k$-algebra with $D_{k}(A)$. If $B$ is

(1) a formal power series ring over $A$ (in a finite number of variables) or

(2) a finite A-algebra, then $D_{k}(B)$ exists.

Proof. Case (1). Put $B=A\left[\left[X_{1}, \cdots, X_{n}\right]\right]$. Let $d_{B / k}: B \rightarrow D$ be the universal prefinite $k$-derivation. Since $D$ can be embedded into a direct product of finite $B$-modules and $I=\left(X_{1}, \cdots, X_{n}\right) B \subset \operatorname{rad}(B), D$ is separated in $I$-adic topology. Thus $D=\sum_{a \in A} B d_{B / k}(a)+\sum_{i} B d_{B / k} X_{i}$. Moreover $D$ is a prefinite $A$-module because for any finite $B$-module $M$ and for any $\nu>0, M / I^{\nu} M$ is a finite $A$-module. Hence there is an A-linear map $f: D_{k}(A)$ $\rightarrow D$ such that $f \circ d_{A / k}=d_{B / k} \circ \phi$ where $d_{A / k}$ is the universal finite $k$-derivation of $A$ and $\phi: A \rightarrow B$ is the natural injection. Therefore $D$ is generated over $B$ by $\operatorname{Im}(f)$ and $d_{B / k} X_{1}, \cdots, d_{B / k} X_{n}$. Hence $D$ is a finite $B$-module.

Case (2). Proof is similar to the case (1).

Remark. In the case (1), we have $D_{k}(B) \simeq\left(D_{k}(A) \bigotimes_{A} B\right) \oplus\left(\oplus_{i} B d X_{i}\right)$, because for any finite $B$-module $M$,

$$
\operatorname{Der}_{k}(B, M) \simeq \operatorname{Der}_{k}(A, M) \oplus \operatorname{Hom}_{B}\left(\oplus B d X_{i}, M\right) .
$$

We now recall some results on extension of fields which are developed in E. G. A. chapter $0_{\mathrm{rv}}$. Let $k_{0} \subset k \subset K$ be fields. The kernel of the natural map $\Omega_{k / k_{0}} \otimes_{k} K \rightarrow \Omega_{K / k_{0}}$ is called the module of imperfection and is denoted by $\Upsilon_{K / k / k_{0}}$. When $k_{0}$ is the prime field, $\Upsilon_{K / k / k_{0}}$ is also denoted by $\Upsilon_{K / k}$. Let $L$ be an extension of $K$. Then we have the following exact sequence:

(***) $\quad 0 \longrightarrow \Upsilon_{K / k / k_{0}} \otimes_{K} L \stackrel{v}{\longrightarrow} \Upsilon_{L / k / k_{0}} \stackrel{u}{\longrightarrow} \Upsilon_{L / K / k_{0}} \stackrel{s}{\longrightarrow} \Upsilon_{L / K / k} \longrightarrow 0$.

The module $Y_{K / k / k_{0}} \otimes_{K} L$ will be also denoted by $Y_{K / R / k_{0}}^{L}$. Obviously the following are equivalent:

(1) $v$ is surjective,

(1) $v$ is bijective,

(2) $u=0$,

(3) $s$ is injective,

(3)' $s$ is bijective.

We say that $k$ is $k_{0}$-admissible for $L / K$ if the conditions above are 
satisfied. When $k_{0}$ is the prime field, we use the word "admissible" instead of $k_{0}$-admissible.

Lemma (0.6) (E. G.A. (0.21.6.5)), Let $k_{0} \subset k \subset k^{\prime} \subset K \subset L \subset M$ be fields. Then

(1) $k^{\prime}$ is $k_{0}$-admissible for $L / K$ if and only if $k$ is $k_{0}$-admissible for $L / K$ and $k^{\prime}$ is $k$-admissible for $L / K$.

(2) $k$ is $k_{0}$-admissible for $M / K$ if and only if $k$ is $k_{0}$-admissible for $M / L$ and $L / K$.

LEMMA (0.7). Let $k \subset K$ be fields and $L$ a finitely generated extension of $K$. Then $\Omega_{L / K}$ and $\gamma_{L / K / k}$ are finite L-module, and we have

$$
\operatorname{rank}_{L} \Omega_{L / K}-\operatorname{rank}_{L} \Upsilon_{L / K / k} \geq \operatorname{tr} . \operatorname{deg}_{K} L .
$$

The equality holds if and only if $k$ is admissible for $L / K$.

Proof is obvious by Cartier's equality (cf. E. G.A. (0.21.7.1)) and the exact sequence $(* * *)$.

Let $K$ be a field and $F=\left(k_{\alpha}\right)_{\alpha \in I}$ a family of subfields of $K$. We say that $F$ is downward directed if the following condition is satisfied:

For any $\alpha, \beta \in I$, there is some $\gamma \in I$ such that $k_{\alpha} \cap k_{\beta} \supset k_{r}$.

LEMma (0.8) (E.G.A. (0.21.8.3)). Let $k_{0} \subset K$ be fields of characteristic $p>0$. Let $\left(k_{\alpha}\right)_{\alpha \in I}$ be a downward directed family of subfields of $K$ containing $k_{0}$. Then the following are equivalent:

(a) $\bigcap_{\alpha} k_{\alpha}\left(K^{p}\right)=k_{0}\left(K^{p}\right)$,

(b) if $L$ is an extension of $K$ with $\operatorname{rank}_{L} \Upsilon_{L / K / k_{0}}<\infty$, then there is some $\alpha \in I$ such that $k_{\alpha}$ is $k_{0}$-admissible for $L / K$,

(b)' for any $x \in K$, there is some $\alpha \in I$ such that $k_{\alpha}$ is $k_{0}$-admissible for $K\left(x^{1 / p}\right) / K$,

(c) the canonical homomorphism $\Omega_{K / k_{0}} \rightarrow \underset{\leftarrow}{\lim _{\alpha}} \Omega_{K / k_{\alpha}}$ is injective.

Remark. Let $k_{0}$ and $K$ be as above. Let $B$ be a $p$-basis of $K$ over $k_{0}$ and $\left\{H_{\alpha}\right\}$ the family of finite subsets of $B$. Put $B_{\alpha}=B \backslash H_{\alpha}$ and $k_{\alpha}=$ $k_{0}\left(K^{p}\right)\left(B_{\alpha}\right)$. Then $\left\{k_{\alpha}\right\}$ is a downward directed family of cofinite subfields of $K$ containing $k_{0}$ which satisfies the conditions of the lemma.

CoRollary (0.9). Suppose that $\left(k_{\alpha}\right)_{\alpha \in I}$ satisfies the conditions of the lemma above. Then, if $L$ is an extension of $K$ with $\operatorname{rank}_{L} \Upsilon_{L / K / k_{0}}<\infty$, we have $\bigcap_{\alpha} k_{\alpha}\left(L^{p}\right)=k_{0}\left(L^{p}\right)$. 
LEMma (0.10) ([2] (30.E)). Let $k$ be a field of characteristic $p>0$ and $\left(k_{\alpha}\right)_{\alpha \in I}$ a downward directed family of subfields of $k$. Put $k_{0}=\bigcap_{\alpha} k_{\alpha}$. Then we have $\bigcap_{\alpha} k_{\alpha}\left(\left(T_{1}, \cdots, T_{r}\right)\right)=k_{0}\left(\left(T_{1}, \cdots, T_{r}\right)\right)$.

\section{$\S 1$. Differential modules of a local ring}

Let $(A, \mathfrak{m})$ be a local ring containing a field $k$. Recall that $k$ is called a quasi-coefficient field of $A$ if $A / \mathfrak{m}$ is formally etale over $k$ (cf. [4]).

Lemma (1.1). Let $(A, \mathfrak{m})$ be a local ring containing a field $k$. Then:

(1) If $A / \mathfrak{m}$ is separable over $k$, there is a quasi-coefficient field of $A$ containing $k$. In particular $A$ has a quasi-coefficient field,

(2) if $k$ is a quasi-coefficient field of $A$, then there is a unique coefficient field of $\hat{A}$ containing $k$,

(3) if $k$ is a quasi-coefficient field of $A$ and $K$ is a coefficient field of $\hat{A}$ containing $k$, then we have $\Omega_{\hat{A} / k} \simeq \Omega_{\hat{A} / K}$.

Proof. (1) and (2) are proved in [4]. (3) is trivial by the following exact sequence: $0=\Omega_{K / k} \otimes{ }_{k} \hat{A} \rightarrow \Omega_{\hat{A} / k} \rightarrow \Omega_{\hat{A} / K} \rightarrow 0$.

Proposition (1.2). Let $k \subset K$ be fields and let $A=K\left[\left[X_{1}, \cdots, X_{n}\right]\right]$. Then the following are equivalent:

(1) $D_{k}(A)$ exists,

(2) $\operatorname{rank}_{K} \Omega_{K / k}<\infty$,

(3) $\operatorname{ch}(k)=0$ and tr. $\operatorname{deg}_{k} K<\infty$, or $\operatorname{ch}(k)=p>0$ and $\left[K: K^{p}(k)\right]<$ $\infty$. Furthermore, if $D_{k}(A)$ exists, it is a free A-module of rank $(n+$ $\left.\operatorname{rank}_{K} \Omega_{K / k}\right)$.

Proof is obvious by (0.4) and (0.5).

Corollary (1.3). Let $A$ be a noetherian complete local ring, $K$ a coefficient field of $A$ and $k$ a subfield of $K$. Then $D_{k}(A)$ exists if and only if $\operatorname{rank}_{K} \Omega_{K / k}<\infty$.

Proposition (1.4). Let $(A, \mathfrak{m}, K)$ be a noetherian local ring containing a field $k$. Assume that

(1) for any cofinite subfield $k^{\prime}$ of $k, D_{k^{\prime}}(A)$ exists, and

(2) $\operatorname{rank} \Upsilon_{K / k}<\infty$.

Then there is a subfield $k_{0}^{\prime}$ of $k$ and a quasi-coefficient field $K_{0}$ of $A$ containing $k_{0}^{\prime}$ such that (1) and (2) continue to hold after replacing $k$ by $k_{0}^{\prime}$ and such that $D_{K_{0}}(A)$ exists. 
Proof. Case (1). $\operatorname{ch}(k)=0$. Since $A$ has a quasi-coefficient field containing $k$ by (1.1), the assertion follows from (0.4), (1).

Case (2). $\quad \operatorname{ch}(k)=p>0$. Let $B$ be a $p$-basis of $k$. By the condition (2) there is a finite subset $F$ of $B$ such that $K$ is separable over $k_{0}^{\prime}=k_{0}\left(B^{\prime}\right)$ where $k_{0}$ is the prime field and $B^{\prime}=B \backslash F$. So $A$ has a quasi-coefficient field $K_{0}$ containing $k_{0}^{\prime}$. Put $k^{\prime}=k^{p}\left(k_{0}^{\prime}\right)=k^{p}\left(B^{\prime}\right)$. Then $D_{k^{\prime}}(A)$ exists because $\left[k: k^{\prime}\right]<\infty$. Since we have $D_{k_{0}^{\prime}}(A)=D_{k^{\prime}}(A)$, the existence of $D_{K_{0}}(A)$ follows from (0.4), (1). If $k_{1}$ is a cofinite subfield of $k_{0}^{\prime}$ then $\left[k^{p}\left(k_{0}^{\prime}\right): k^{p}\left(k_{1}\right)\right]<$ $\infty$, hence $\left[k: k^{p}\left(k_{1}\right)\right]<\infty$ and $D_{k^{p}\left(k_{1}\right)}(A)=D_{k_{1}}(A)$ exists.

Let $A$ be an integral domain and $M$ an $A$-module. We put $\operatorname{rank}_{A} M$ $=\operatorname{rank}_{Q(A)} M \otimes_{A} Q(A)$, where $Q(A)$ denotes the quotient field of $A$. We will investigate the rank of differential module of a local domain.

Proposition (1.5). Let $K$ be a field of characteristic $p>0$ and $k a$ subfield of $K$ such that $\operatorname{rank}_{K} \Omega_{K / k}<\infty$. Let $A=K\left[\left[X_{1}, \cdots, X_{n}\right]\right], P$ a prime ideal of $A$ and $R=A / P$. Then there is a k-subalgebra $R_{0}$ of $R$ such that $D_{k}(R) \simeq \Omega_{R / R_{0}}$.

Proof. By the normalization theorem, there is a $k$-subalgebra $R_{1}=$ $K\left[\left[T_{1}, \cdots, T_{d}\right]\right]$ of $R$ such that $R$ is a finite $R_{1}$-algebra $(d=\operatorname{dim} R)$. Put $R_{0}=k\left(K^{p}\right)\left[\left[T_{1}^{p}, \cdots, T_{d}^{p}\right]\right]$. Then $R$ is a finite $R_{0}$-algebra because $\operatorname{rank}_{K} \Omega_{K / k}$ $<\infty$. So $\Omega_{R / R_{0}}$ is a finite $R$-module. Let $N$ be an arbitrary finite $R$ module. Since $N$ is separated in $m_{R}$-adic topology $\left(m_{R}\right.$ denotes the maximal ideal of $R$ ) and $T_{i} \in m_{R}$, any derivation of $R$ into $N$ over $k$ vanishes on $R_{0}$, hence we have $\operatorname{Hom}_{R}\left(D_{k}(R), N\right) \simeq \operatorname{Der}_{k}(R, N) \simeq \operatorname{Hom}_{R}\left(\Omega_{R / R_{0}}, N\right)$. This shows that $D_{k}(R) \simeq \Omega_{R / R_{0}}$ by the universal mapping property of $D_{k}(R)$.

Remark. If $[K: k]<\infty$, we can replace $R_{0}$ by $k\left[\left[T_{0}^{p}, \cdots, T_{d}^{p}\right]\right]$.

Corollary (1.6). Let $M=Q(R)$ and $M_{0}=Q\left(R_{0}\right)$. Then we have

$$
\operatorname{rank}_{R} D_{k}(R)=\operatorname{rank}_{M} \Omega_{M / M_{0}} \text {. }
$$

Corollary (1.7). Put $M_{1}=Q\left(R_{1}\right)$. Then the following are equivalent:

(1) $M_{0}$ is admissible for $M / M_{1}$,

(2) $\operatorname{rank}_{R} D_{k}(R)=\operatorname{dim} R+\operatorname{rank}_{K} \Omega_{K / k}$.

Proof. Let $B$ be a $p$-basis of $K$ over $k$. Then $B \cup\left\{T_{1}, \cdots, T_{d}\right\}$ is a $p$-basis of $R_{1}$ over $R_{0}$ and hence we have $\operatorname{rank}_{M_{1}} \Omega_{M_{1} / M_{0}}=d+\operatorname{rank}_{K} \Omega_{K / k}$. Consider the following exact sequence:

$$
0 \rightarrow \Upsilon_{M / M_{1} / M_{0}} \rightarrow \Omega_{M_{1} / M_{0}} \otimes_{M_{1}} M \rightarrow \Omega_{M / M_{0}} \rightarrow \Omega_{M / M_{1}} \rightarrow 0
$$


Since we have $\left[M: M_{1}\right]<\infty$, the condition (1) is equivalent to the following by (0.7):

$$
\operatorname{rank}_{M_{1}} \Omega_{M_{1} / M_{0}}=\operatorname{rank}_{M} \Omega_{M / M_{0}} .
$$

Clearly (1)' is equivalent to (2) by (1.6).

The following proposition is an analogy of E.G.A. (0.21.9.8).

Proposition (1.8). Let $k, K$ and $R$ be as in Proposition (1.5). Assume moreover that $\operatorname{rank}_{K} Y_{K / k}<\infty$. Then there is a cofinite subfield $k^{\prime}$ of $k$ which satisfies the following: for any cofinite subfield $k^{\prime \prime}$ of $k^{\prime}$, we have

$$
\operatorname{rank}_{R} D_{k^{\prime \prime}}(R)=\operatorname{dim} R+\operatorname{rank}_{K} \Omega_{K / k^{\prime \prime}} .
$$

Proof. Let $R_{1}=K\left[\left[T_{1}, \cdots, T_{d}\right]\right](d=\operatorname{dim} R)$ be a subring of $R$ such that $R$ is a finite $R_{1}$-algebra, $M=Q(R)$ and $M_{1}=Q\left(R_{1}\right)$. Let $\left(k_{\alpha}\right)_{\alpha \in I}$ be a downward directed family of cofinite subfields of $k$ with $\cap_{\alpha} k_{\alpha}=k^{p}$. Since $\operatorname{rank} \Upsilon_{K / k}<\infty$ we have $\bigcap_{\alpha} k_{\alpha}\left(K^{p}\right)=K^{p}$ by (0.9). We put $M_{\alpha}=k_{\alpha}\left(K^{p}\right)\left(\left(\Upsilon_{1}^{p}\right.\right.$, $\left.\left.\cdots, \Upsilon_{d}^{p}\right)\right)$ for each $\alpha$. Then we have by $(0.10) \cap_{\alpha} M_{\alpha}=K^{p}\left(\left(\Upsilon_{1}^{p}, \cdots, \Upsilon_{d}^{p}\right)\right)=\left(M_{1}^{p}\right)$. Since $M$ is a finite extension of $M_{1}$ we have rank $\Upsilon_{M / M_{1}}<\infty$. Hence there is some $\alpha \in I$ such that $M_{\alpha}$ is admissible for $M / M_{1}$ by (0.8). Moreover if $k^{\prime \prime}$ is a cofinite subfield of $k_{\alpha}$, the field $M^{\prime \prime}=k^{\prime \prime}\left(K^{p}\right)\left(\left(\Upsilon_{1}^{p}, \cdots, \Upsilon_{d}^{p}\right)\right)$ is also admissible for $M / M_{1}$ by (0.6), (1). This means by (1.7) that $\operatorname{rank} D_{k^{\prime \prime}}(R)$ $=\operatorname{dim} R+\operatorname{rank} \Omega_{K / k^{\prime \prime}}$. Hence we can take this $k_{\alpha}$ for $k^{\prime}$.

Lemma (1.9). Let $A$ be a noetherian local domain and $k$ a subfield. Assume that $D_{k}(A)$ exists and that $A$ is analytically unramified. Then, for each $P \in \operatorname{Ass}(\hat{A})$, we have $\operatorname{rank}_{A} D_{k}(A)=\operatorname{rank}_{\hat{A} / P} D_{k}(\hat{A} / P)$.

Proof. By (0.4), (3), we have the following exact sequence:

$$
\left(P / P^{2}\right) \otimes_{\hat{A}} k(P) \rightarrow D_{K}(\hat{A}) \otimes_{\hat{A}} k(P) \rightarrow D_{k}(\hat{A} / P) \otimes_{\hat{A} / P} k(P) \rightarrow 0 .
$$

This shows that $D_{k}(\hat{A}) \otimes_{\hat{A}^{k}}(P) \simeq D_{k}(\hat{A} / P) \otimes_{\hat{A} / P} k(P)$ because $\hat{A}$ is reduced and so $P \hat{A}_{p}=0$ and $\kappa(P)=\hat{A}_{P}$. Therefore we have

$$
\operatorname{rank}_{A} D_{k}(A)=\operatorname{rank}_{k(P)} D_{k}(\hat{A}) \otimes_{\hat{A} k}(P)=\operatorname{rank}_{\hat{A} / P} D_{k}(\hat{A} / P) .
$$

Theorem (1.10). Let $R$ be a noetherian local domain, $K$ a quasi-coefficient field of $R$ and $k$ a subfield of $K$. Assume that

(1) for any cofinite subfield $k_{1}$ of $k, D_{k_{1}}(R)$ exists,

(2) $\operatorname{rank}_{K} \Upsilon_{K / k}<\infty$, and

(3) $R$ is analytically unramified. 
Then there is a cofinite subfield $k^{\prime}$ of $k$ which satisfies the following: for any cofinite subfield $k^{\prime \prime}$ of $k^{\prime}$, we have $\operatorname{rank}_{R} D_{k^{\prime \prime}}(R)=\operatorname{dim} R+\operatorname{rank}_{K} \Omega_{K / k^{\prime \prime}}$.

Proof. Let $K^{*}$ be a coefficient field of $\hat{R}$ containing $K$. Then, if $K^{\prime}$ is a subfield of $K$, we have $\Upsilon_{K / K^{\prime}}^{K^{*}} \simeq \Upsilon_{K^{*} / K^{\prime}}$ and $\Omega_{K / K^{\prime}} \otimes_{K} K^{*} \simeq \Omega_{K^{*} / K^{\prime}}$. In particular we have $\operatorname{rank}_{K^{*}} \Upsilon_{K^{*} / k}<\infty$. Next let $k_{1}$ be a cofinite subfield of $k$ and $P \in \operatorname{Ass}(\hat{R})$ be such that $\operatorname{dim}(R / P)=\operatorname{dim} \hat{R}=\operatorname{dim} R$. Then we have $\operatorname{rank}_{R} D_{k_{1}}(R)=\operatorname{rank}_{\hat{R} / P} D_{k_{1}}(\hat{R} / P)$ by (1.9). Therefore we get the assertion by (1.8) with $\left(k, K^{*}, \hat{R} / P\right)$ for $(k, K, R)$.

CoRollary (1.11). Under the assumption of the theorem, $\hat{R}$ is equidimensional and hence $R$ is universally catenary.

Proof. Let $\operatorname{Ass}(\hat{R})=\left\{P_{1}, \cdots, P_{r}\right\}$. Then, by the theorem, there is a cofinite subfield $k^{\prime}$ of $k$ such that $\operatorname{rank}_{\hat{R} / P_{i}} D_{k^{\prime}}\left(\hat{R} / P_{i}\right)=\operatorname{dim}\left(\hat{R} / P_{i}\right)+\operatorname{rank}_{K} \Omega_{K / k^{\prime}}$ for each $i$. This shows that $\operatorname{dim}\left(\hat{R} / P_{i}\right)$ is independent on $i$ because $\operatorname{rank}_{R} D_{k^{\prime}}(R)=\operatorname{rank}_{\hat{R} / P_{i}} D_{k^{\prime}}\left(R / P_{i}\right)$ by (1.9). The last assertion follows from this and E.G.A. (IV. 7.1).

We now give a criterion of the regularity of a local ring with universal finite module of differentials. We use the following lemma.

LEMma (1.12). Let $(A, \mathfrak{m})$ be a regular local ring and $I$ an ideal of $A$. Let $\alpha:\left(I / I^{2}\right) \otimes_{A} A / \mathfrak{m} \rightarrow\left(\mathfrak{m} / \mathfrak{m}^{2}\right) \otimes_{A} A / \mathfrak{m}$ be the natural map. Then

(1) $\operatorname{rank}_{A / \mathrm{m}} \operatorname{Im}(\alpha) \leq \mathrm{ht} I$.

(2) The following are equivalent:

(a) $A / I$ is a regular local ring,

(b) $\alpha$ is injective,

(c) $\operatorname{rank}_{\mathrm{A} / \mathrm{m}} \operatorname{Im}(\alpha)=\mathrm{ht} I$.

Proof. Note that $A / I$ is regular if and only if $I$ is generated by a subset of a regular system of parameters of $A$. The assertions follow from this fact.

Proposition (1.13). Let $k \subset K$ be fields, $A=K\left[\left[X_{1}, \cdots, X_{n}\right]\right], I$ an ideal of $A$ such that $I=\sqrt{I}$ and $P \in \operatorname{Spec}(A)$ with $P \supset I$. We put $R=$ $A / I$ and $\mathfrak{p}=P / I$. Assume that $D_{k}(R)$ exists and that we have

$$
\operatorname{rank}_{R / \mathfrak{q}} D_{k}(R / \mathfrak{q})=\operatorname{dim}(R / \mathfrak{q})+\operatorname{rank}_{K} \Omega_{K / k}
$$

for each $\mathfrak{q} \in \operatorname{Ass}(R)$. Then:

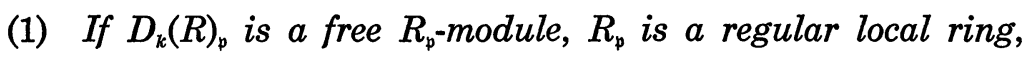


(2) conversely, if $R_{\mathfrak{p}}$ is regular and we have

$$
\operatorname{rank}_{R / \mathfrak{p}} D_{k}(R / \mathfrak{p})=\operatorname{dim}(R / \mathfrak{p})+\operatorname{rank}_{K} \Omega_{K / k},
$$

then $D_{k}(R)_{\mathfrak{p}}$ is a free $R_{\mathfrak{p}}$-module.

Proof. Take $Q \in \operatorname{Ass}_{A}(A / I)$ such that $Q \subset P$ and ht $I A_{p}=$ ht $Q A_{p}$ $(=$ ht $Q$ ). Then $\mathfrak{q}=Q / I$ is a minimal prime ideal of $R$ with $\mathfrak{p} \supset \mathfrak{q}$. Since $R$ is reduced we have $D_{k}(R) \otimes_{R} k(\mathfrak{q}) \simeq D_{k}(R / \mathfrak{q}) \otimes_{R / \mathfrak{p}} k(\mathfrak{q})$. Now consider the following commutative diagram with exact rows:

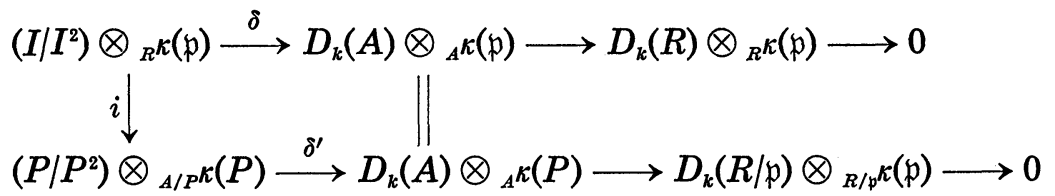

Then, if $D_{k}(R)_{\mathfrak{p}}$ is $R_{\mathfrak{p}}$-free, we have $\operatorname{rank}_{k(\mathfrak{p})} D_{k}(R) \otimes_{R} \kappa(\mathfrak{p})=\operatorname{rank}_{\kappa(\mathfrak{q})} D_{k}(R)$ $\otimes_{R} k(\mathfrak{q})=\operatorname{rank}_{R / \mathfrak{q}} D_{k}(R / \mathfrak{q})=\operatorname{dim}(R / \mathfrak{q})+\operatorname{rank}_{K} \Omega_{K / k}$. Thus we have $\operatorname{rank}_{k(\mathfrak{p})} \operatorname{Im}(i)$ $\geq \operatorname{rank}_{\mathbf{k}(\mathcal{p})} \operatorname{Im}(\delta)=\operatorname{dim} A+\operatorname{rank}_{K} \Omega_{K / k}-\left(\operatorname{dim}(R / \mathfrak{q})+\operatorname{rank}_{K} \Omega_{K / k}\right)=\operatorname{ht} Q=$ ht $I A_{p}$. This means that $R_{p}=A_{p} / I A_{p}$ is regular by (1.12). Hence we have proved (1).

To prove (2), note that if the equality in (2) holds, then $\delta^{\prime}$ is injective. Hence $\delta$ is injective if and only if $i$ is injective. This last condition is equivalent to that $R_{\mathfrak{p}}$ is regular by (1.12). Therefore if $R_{\mathfrak{p}}$ is regular, we have rank $D_{k}(R) \otimes_{R} k(\mathfrak{p})=\operatorname{dim} A+\operatorname{rank} \Omega_{K / k}-\mathrm{ht} I A_{p} . \quad$ On the other hand we have $\operatorname{rank} D_{k}(R) \otimes \kappa(\mathfrak{p}) \geq \operatorname{rank} D_{k}(R)_{\mathfrak{p}} \geq \operatorname{dim} A+\operatorname{rank} \Omega_{K / k}-\mathrm{ht} I A_{p}$, where the first inequality is obvious and the second follows from the exact sequence: $\left(I / I^{2}\right) \otimes_{R} R_{\mathfrak{p}} \rightarrow D_{k}(A) \otimes{ }_{A} R_{\mathfrak{p}} \rightarrow D_{k}(R)_{\mathfrak{p}} \rightarrow 0$ and the regularity of $R_{\mathfrak{p}}$. Hence we have $\operatorname{rank} D_{k}(R)_{\mathfrak{p}} \otimes_{R_{\mathfrak{p}}} k(\mathfrak{p})=\operatorname{rank} D_{k}(R)_{\mathfrak{p}}$ and this shows that $D_{k}(R)_{\mathfrak{p}}$ is a free $R_{\mathrm{p}}$-module.

The following theorem is a corollary of the proposition above.

TheOREM (1.14). Let $R$ be a noetherian local domain containing a field $k$ and $\mathfrak{p} \in \operatorname{Spec}(R)$. Assume that

(1) $R$ has a quasi-coefficient field $K$ containing $k$,

(2) $R$ is analytically unramified,

(3) for any cofinite subfield $k^{\prime}$ of $k, D_{k^{\prime}}(R)$ exists, and

(4) $\operatorname{rank}_{R} D_{k}(R)=\operatorname{dim} R+\operatorname{rank}_{K} \Omega_{K / k}$.

Then, if $D_{k}(R)_{\mathfrak{p}}$ is a free $R_{\mathfrak{p}}$-module, $R_{\mathfrak{p}}$ is a regular local ring.

Proof. Let $\mathfrak{q}$ be a prime ideal of $\hat{R}$ lying over $\mathfrak{p}$. Then $D_{k}(\dot{R})_{\mathfrak{q}}=$ 
$D_{k}(R)_{p} \otimes_{R_{p}} \hat{R}_{q}$ is a free $\hat{R}_{q}$-module. Let $K^{*}$ be a coefficient field of $\hat{R}$ containing $K . \quad \hat{R}$ is equidimensional by (1.11). So, for each $\mathfrak{q}_{\imath} \in \operatorname{Ass}(\hat{R})$, we have $\operatorname{rank}_{\hat{R} / q_{i}} D_{k}\left(\hat{R} / \mathfrak{q}_{i}\right)=\operatorname{rank}_{R} D_{k}(R)=\operatorname{dim} R+\operatorname{rank}_{K} \Omega_{K / k}=\operatorname{dim}\left(\hat{R} / \mathfrak{q}_{i}\right)+$ $\operatorname{rank}_{R^{*}} \Omega_{K^{*} / k}$ by (1.9). Hence $\hat{R}_{q}$ is regular by $(1,13)$. Since $\hat{R}_{q}$ is faithfully flat over $R_{p}, R_{p}$ is regular.

ExAmples. Let $k$ be a field and $R$ a noetherian $k$-algebra with $D_{k}(R)$. When $\operatorname{ch}(k)=0$, Scheja and Storch proved the following (cf. [8]):

let $\mathfrak{p}$ be a prime ideal of $R$, then $R_{\mathfrak{p}}$ is regular if and only if $D_{k}(R)_{\mathfrak{p}}$ is a free $R_{p}$-module.

When $\operatorname{ch}(k)=p>0$, the following examples (1) and (2) show that the above result must be modified as in (1.14).

(1) Put $R=k[X] /\left(X^{p}\right)$. Then $R$ is an artinian local ring and is not regular. On the other hand $D_{k}(R)$ exists and is a free $R$-module of rank 1. For the unique prime ideal $m_{R}$ of $R$, we have $\operatorname{rank}_{R / m_{R}} D_{k}\left(R / m_{R}\right)=0$.

(2) Put $A=k[[X, Y]]$ and suppose $k \neq k^{p}$. If $a \in k-k^{p}$ then $a X^{p}+$ $Y^{p}$ is an irreducible element of $A$. Put $R=A /\left(a X^{p}+Y^{p}\right)$. Then $D_{k}(R)$ is a free $R$-module of rank 2. The ring $R$ is a local domain of dimension 1 and is not regular.

(3) Assume moreover that $\left[k: k^{p}\right]=\infty$. Let $A=k^{p}[[T]][k]$. Take $u \in k[[T]]-A$ and put $a=u^{p}$. Then $R=A[X] /\left(X^{p}-a\right)$ is a local domain of dimension 1. $\hat{R}=\hat{A}[X] /(X-u)^{p}$ is not reduced. It is easy to see that for any cofinite subfield $k^{\prime}$ of $k$ containing $k^{p}, D_{k^{\prime}}(R)$ exists and $D_{k^{\prime}}(R)=$ $\left(\Omega_{k / k^{\prime}} \otimes_{k} R\right) \oplus R d T \oplus R d X$. Hence we have $\operatorname{rank}_{R} D_{k^{\prime}}(R)=\operatorname{rank}_{k} \Omega_{k / k^{\prime}}+2$. Thus the assumption (4) of Theorem (1.14) is essential.

\section{§2. Formal fibres and regular loci}

THEOREM (2.1). Let $k$ be a field of characteristic $p>0$ and let $A$ be $a$ noetherian k-algebra. Assume that

(1) A is a locally Nagata ring,

(2) for any cofinite subfield $k^{\prime}$ of $k, D_{k^{\prime}}(A)$ exists, and

(3) for any maximal ideal $\mathfrak{m}$ of $A$, we have $\operatorname{rank}_{x(\mathrm{~m})} \Upsilon_{x(\mathrm{~m}) / k}<\infty$. Then $A$ is a universally catenary G-ring.

Proof. Note that $A$ is universally catenary if and only if for any maximal ideal $\mathfrak{m}$ of $A$ and any prime ideal $\mathfrak{p}$ contained in $\mathfrak{m}, A_{\mathfrak{m}} / \mathfrak{p} A_{\mathrm{m}}$ is universally catenary. Hence we can assume by $(0.3)$ and (0.4) that $A$ is a local domain. Moreover we can assume that $k$ is contained in a quasi- 
coefficient field of $A$ by the conditions (2), (3) and Proposition (1.4). The local ring $A$ is analytically unramified by (1) (cf. [2] Theorem 70). Hence $A$ is universally catenary by (1.11)). To prove that $A$ is a $G$-ring, we can again localize at a maximal ideal and assume that $A$ is a local ring with maximal ideal $\mathrm{m}$. Then we have the following lemma.

Lemma (2.2) ([2]. (33. E)). Let $R$ be a noetherian local ring. Then $R$ is a G-ring if and only if, for any finite integral $R$-algebra $S$ and for any prime ideal $Q$ of $\hat{S}$ such that $Q \cap S=(0), \hat{S}_{Q}$ is a regular local ring.

Let $B$ be a finite integral $A$-algebra and $Q$ a prime ideal of $\hat{B}$ such that $Q \cap B=(0)$. Let $\mathfrak{n}^{*}$ be a maximal ideal of $\hat{B}$ containing $Q$ and put $\mathfrak{n}=\mathfrak{n}^{*} \cap B$. Then $\mathfrak{n}$ is a maximal ideal of $B$ and we have $\hat{B}_{\mathfrak{n}^{*}}=\left(B_{\mathfrak{n}}\right)^{\wedge}$. Since $B$ is a finite $A$-algebra, $B$ and $k$ satisfies (1), (2) and (3). So replacing $A$ by $B_{n}$, we have only to prove that when $A$ is a local domain, $\hat{A}_{Q}$ is a regular local ring whenever $Q$ is a prime ideal of $\hat{A}$ with $Q \cap A=$ (0). Again we can assume that $k$ is contained in a quasi-coefficient field of $A$. Then by (1.10), there is a cofinite subfield $k^{\prime}$ of $k$ such that $\operatorname{rank}_{A} D_{k^{\prime}}(A)=\operatorname{dim} A+\operatorname{rank}_{\kappa(\mathfrak{m})} \Omega_{\kappa(\mathfrak{m}) / k^{\prime}}$. Since $\hat{A}$ is reduced and $D_{k^{\prime}}(\hat{A})_{Q}=$ $D_{k^{\prime}}(A) \otimes_{A} Q(A) \otimes_{Q(A)} \hat{A}_{Q}$ is a free $\hat{A}_{Q}$-module, $\hat{A}_{Q}$ is a regular local ring by (1.13), (1.9) and (1.11).

THEOREM (2.3). In addition to the assumption in (2.1), assume that $k$ is a perfect field. Then $A$ is an excellent ring.

Proof. We have only to prove that $A$ is $J-2$, i.e., that for any finite $A$-algebra $A^{\prime}, \operatorname{Reg}\left(A^{\prime}\right)$ is an open subset of $\operatorname{Spec}\left(A^{\prime}\right)$. To prove this, it is sufficient to show the following (cf. [2] (32. A) Lemma 1): $\operatorname{Reg}\left(A^{\prime} / \mathfrak{p}\right)$ contains a non-empty open subset of $\operatorname{Spec}\left(A^{\prime} / \mathfrak{p}\right)$ for each $\mathfrak{p} \in \operatorname{Spec}\left(A^{\prime}\right)$. Since $A^{\prime} / \mathfrak{p}$ satisfies the same condition as $A$, the problem is reduced to showing that when $A$ is an integral domain $\operatorname{Reg}(A)$ contains a non-empty open subset of $\operatorname{Spec}(A)$. First we prove that the following equality holds for each maximal ideal $\mathfrak{m}$ of $A:(*) \operatorname{rank} D_{k}\left(A_{\mathfrak{m}}\right)=\operatorname{dim} A_{\mathfrak{m}}+\operatorname{rank} \Omega_{k(\mathfrak{m}) / k}$. In fact $A_{\mathrm{m}}$ is analytically unramified because it is a Nagata ring. Furthermore since $k$ is perfect, $A_{\mathrm{m}}$ has a quasi-coefficient field containing $k$ by (1.1), (1), and $k=k^{p}$. Hence we get the equality by (1.10). Now we prove the set $U=\left\{\mathfrak{p} \in \operatorname{Spec}(A) \mid D_{k}(A)_{\mathfrak{p}}\right.$ is a free $A_{\mathfrak{p}}$-module $\}$ is contained in $\operatorname{Reg}(A)$. Let $\mathfrak{p} \in U$. Take a maximal ideal $\mathfrak{m}$ containing $\mathfrak{p}$. Then $D_{k}(A)_{\mathfrak{p}}$ $=\left(D_{k}(A) \otimes_{A} A_{\mathfrak{m}}\right)_{\mathfrak{p}}=D_{k}\left(A_{\mathfrak{n}}\right)_{\mathfrak{p}}$ is a free $A_{\mathfrak{p}}$-module and $(*)$ holds for $\mathfrak{m}$. 
Hence $A_{\mathfrak{p}}=\left(A_{\mathrm{m}}\right)_{\mathfrak{p}}$ is regular by (1.14).

Lastly we will prove Matsumura's theorem ([5] Theorem 15) in a slightly different way. Let $R$ be a regular ring and $\mathrm{P}$ a prime ideal of height $r$. Matsumura defined the following condition (cf. [5]):

$(W J)$ There are some derivations $d_{1}, \cdots, d_{r} \in \operatorname{Der}(R)$ and some elements $f_{1}, \cdots, f_{r} \in \mathrm{P}$ such that $\operatorname{det}\left(d_{i} f_{j}\right) \notin \mathrm{P}$.

Lemma (2.4) ([5] Theorem 14). Let $A$ be a regular ring. Then $A$ is excellent if the following is satisfied:

For any $n \geq 0$ and for any $P \in \operatorname{Spec}\left(A\left[X_{1}, \cdots, X_{n}\right]\right)$ such that $A[X] / P$ is a finite A-algebra, (WJ) holds at $P$.

TheOREM (2.5) (Matsumura). Let $A$ be a regular ring containing $a$ field $k$ of characteristic $p>0$. Assume that

(1) $A$ is a locally Nagata ring,

(2) for any cofinite subfield $k^{\prime}$ of $k, D_{k^{\prime}}(A)$ exists, and

(3) for any maximal ideal $\mathfrak{m}$ of $A$, we have $\operatorname{rank}_{\boldsymbol{k}(\mathrm{m})} \Upsilon_{\boldsymbol{k}(\mathrm{m}) / k}<\infty$. Then $A$ is an excellent ring.

Proof. Put $A_{n}=A\left[X_{1}, \cdots, X_{n}\right]$. Let $P \in \operatorname{Spec}\left(A_{n}\right)$ such that $B=A_{n} / P$ is a finite $A$-algebra. We prove that $(W J)$ holds at $P$. Let $\mathfrak{n}$ be a maximal ideal of $A_{n}$ containing $P$ and put $\mathfrak{m}=\mathfrak{n} \cap A$. Then $\mathfrak{m}$ is a maximal ideal of $A$ since $B$ is a finite $A$-algebra. Replacing $A$ by $A_{\mathrm{m}}$ and $A_{n}$ by $\left(A_{n}\right)_{\mathrm{m}}$, we can assume that $A$ is a regular local ring. Let $B$ be a $p$-basis of $k$ and let $\left\{\boldsymbol{F}_{\alpha}\right\}_{\alpha \in I}$ be the family of finite subsets of $\boldsymbol{B}$. Put $k_{\alpha}=k^{p}\left(\boldsymbol{B}_{\alpha}\right)$ and $k_{\alpha}^{\prime}=k_{0}\left(\boldsymbol{B}_{\alpha}\right)$ where $k_{0}$ is the prime field and $\boldsymbol{B}_{\alpha}=\boldsymbol{B} \backslash \boldsymbol{F}_{\alpha}$. Then $\left(k_{\alpha}\right)_{\alpha \in I}$ (resp. $\left.\left(k_{\alpha}^{\prime}\right)_{\alpha \in I}\right)$ is a downward directed family of cofinite subfields of $k$ (resp. $k_{0}(B)$ ) with $\bigcap_{\alpha} k_{\alpha}=k^{p}$ (resp. $\left.\bigcap_{\alpha} k_{\alpha}^{\prime}=\left(k_{0}(B)\right)^{p}\right)$. Note that $D_{k_{\alpha}}(A)$ exists for each $\alpha$ by (2) and that $D_{k_{\alpha}}(A)=D_{k_{\alpha}^{\prime}}(A)$. Moreover $D_{k_{\alpha}}(B)$ exists and $D_{k_{\alpha}}(B)=D_{k_{\alpha}^{\prime}}(B)$. There is some $\alpha \in I$ such that $k(\mathfrak{m})$ is separable over $k_{\alpha}^{\prime}$ by (3), and hence $A$ has a quasi-coefficient field $K_{A}$ containing $k_{\alpha}^{\prime}$. Replacing $k$ by $k_{\alpha}^{\prime}$, we can assume that $k \subset K_{A}$. Similarly we can assume that $k$ is contained in a quasi-coefficient field of $B_{\mathfrak{n}}$ because $[\kappa(\mathfrak{n}): \kappa(\mathfrak{m})]$ $<\infty$ and hence we have $\operatorname{rank}_{k(\mathfrak{n})} r_{k(\mathfrak{n}) / k}<\infty$. Since $\operatorname{rank}_{k(\mathfrak{n})} \gamma_{k(\mathfrak{n}) / \kappa(\mathfrak{m})}<\infty$ there is some $\beta \in I$ such that $k_{\beta}$ is admissible for $\kappa(\mathfrak{n}) / \kappa(\mathfrak{m})$ by $(0.8)$ and (0.9). Consider the following exact sequence:

$$
0 \rightarrow Y_{\kappa(\mathfrak{n}) / k(\mathfrak{m}) / k_{\beta}} \rightarrow \Omega_{x(\mathfrak{m}) / k_{\beta}} \otimes_{\kappa(\mathfrak{m})} \kappa(\mathfrak{n}) \rightarrow \Omega_{\kappa(\mathfrak{n}) / k_{\beta}} \rightarrow \Omega_{\kappa(\mathfrak{n}) / \kappa(\mathfrak{m})} \rightarrow 0 .
$$

We have $\operatorname{rank}_{\boldsymbol{k}(\mathfrak{m})} \Omega_{\kappa(\mathfrak{m}) / k_{\beta}}<\infty$ and $\operatorname{rank}_{\kappa(\mathfrak{n})} \Omega_{\kappa(\mathfrak{n}) / k_{\beta}}<\infty$ because $D_{k_{\beta}^{\prime}}(A)$ and 
$D_{k_{\beta}}\left(B_{\mathrm{n}}\right)$ exist. Thus we have by (0.7) $\operatorname{rank}_{\kappa(\mathrm{m})} \Omega_{\kappa(\mathrm{m}) / k_{\beta}}-\operatorname{rank}_{k^{(\mathrm{n})}} \Omega_{\left.\varepsilon^{(\mathrm{n})}\right) / k_{\beta}}=$ $\operatorname{rank}_{\kappa(\mathrm{n})} \Omega_{\kappa(\mathrm{n}) / \kappa(\mathrm{m})}-\operatorname{rank}_{\kappa(\mathrm{n})} \gamma_{k(\mathrm{n}) / \kappa(\mathrm{m}) / k_{\beta}}=0$. On the other hand, since $B_{\mathrm{n}}$ is a Nagata ring and hence analytically unramified, there is some $\gamma \in I$ such that $\operatorname{rank}_{B_{\mathrm{n}}} D_{k_{r}}\left(B_{\mathrm{n}}\right)=\operatorname{dim} B_{\mathrm{n}}+\operatorname{rank}_{x(\mathrm{n})} \Omega_{x(\mathrm{n}) / k_{r}}$ by (1.10). We can assume that $k=k_{\beta}=k_{r}$. Put $D=\left(D_{k}(A) \otimes_{A} B\right) \oplus\left(\oplus_{i=1}^{n} B d X_{i}\right)$. There are natural $B$-homomorphism $P / P^{2} \rightarrow \Omega_{A_{n} / k} \otimes_{A_{n}} B=\left(\Omega_{A / k} \otimes_{A} B\right) \oplus\left(\oplus_{i=1}^{n} B d X_{i}\right)$ and $\Omega_{A / k}$ $\rightarrow D_{k}(A)$. Hence we get a $B$-homomorphism $\delta: P / P^{2} \rightarrow D$. We have also a $B$-homomorphism $u: D \rightarrow D_{k}(B)$ defined by the natural map $D_{k}(A) \otimes_{A} B$ $\rightarrow D_{k}(B)$ and $d X_{i} \mapsto d x_{i}\left(x_{i}=X_{i} \bmod P\right)$. Thus we get the following sequence of $B$-homomorphism:

$$
P / P^{2} \stackrel{\delta}{\longrightarrow} D \stackrel{u}{\longrightarrow} D_{k}(B) \longrightarrow 0 \text {. }
$$

Now we prove that $(*)$ is exact. Let $M$ be an arbitrary finite $B$-module. Then $M$ is a finite $A$-module. Hence $\operatorname{Hom}_{B}(D, M) \simeq \operatorname{Hom}_{B}\left(D_{k}(A) \otimes_{A} B, M\right) \oplus$ $\operatorname{Hom}_{B}\left(\oplus_{i=1}^{n} B d X_{i}, M\right) \simeq \operatorname{Der}_{k}(A, M) \oplus \operatorname{Hom}_{A_{n}}\left(\oplus_{i=1}^{n} A_{n} d X_{i}, M\right) \simeq \operatorname{Der}_{k}\left(A_{n}, M\right)$. Thus the following sequence is exact:

$$
0 \longrightarrow \operatorname{Hom}_{B}\left(D_{k}(B), M\right) \longrightarrow \operatorname{Hom}_{B}(D, M) \longrightarrow \operatorname{Hom}_{B}\left(P / P^{2}, M\right) \text {. }
$$

Therefore $(*)$ is exact. Tensoring $(*)$ with $\kappa(P)=Q(B)$, we have the following exact sequence:

$$
P / P^{2} \otimes_{B} \kappa(P) \stackrel{\Delta}{\longrightarrow}\left(D_{k}(A) \otimes_{A} k(P)\right) \oplus\left(\bigoplus_{i=1}^{n} k(P) d X_{i}\right) \longrightarrow D_{k}(B) \otimes_{B} k(P) \longrightarrow 0 \text {. }
$$

Since $A$ is a complete regular local ring, $D_{k}(\hat{A})=D_{k}(A) \otimes_{A} \hat{A}$ is a free $A$ module of $\operatorname{rank}\left(\operatorname{dim} \hat{A}+\operatorname{rank} \Omega_{k(\mathrm{~m}) / k}\right)$ by (1.2). Hence $D_{k}(A)$ is $A$-free by [2] (4. E) and we have $\operatorname{rank} \operatorname{Im}(\Delta)=\operatorname{rank} D_{k}(A)+n-\operatorname{rank} D_{k}(B)=\operatorname{dim} A$ $+\operatorname{rank} \Omega_{\varepsilon(\mathrm{m}) / k}+n-\left(\operatorname{rank} \Omega_{c(\mathrm{n}) / k}+\operatorname{dim} B_{\mathrm{n}}\right)=\operatorname{ht} P$. This means that $(W J)$ hold at $P$.

Corollary (2.6) (cf. [5] and [9]). Let $k$ be a field. Then the ring $k\left[X_{1}, \cdots, X_{m}\right]\left[\left[Y_{1}, \cdots, Y_{n}\right]\right]$ is excellent.

Remark. Let $k$ be a field and $A$ a noetherian $k$-algebra with $D_{k}(A)$. When the characteristic of $k$ is zero, then $A$ is excellent (cf. [8]). When the characteristic of $k$ is $p>0, A$ is not necessary excellent. For instance, the ring $A=k^{p}[[T]][k]$ is not excellent if $\left[k: k^{p}\right]=\infty$ (cf. [2] (34. B)), while $D_{k}(A)$ exists and is a free $A$-module of rank 1. (cf. also $\S 1$, Example (3).) 


\section{REFERENCES}

[1] Marot, J., Sur les anneaux universellement japonais, C. R. Acad. Sci. Paris, 277 (1973), 1029-1031.

[2] Matsumura, H., Commutative Algebra, Benjamin, New York, 1970.

[3] — Formal power series rings over polynomial rings I, in "Number Theory, Algebraic Geometry, and Commutative Algebra" (in honor of Y. Akizuki), Kinokuniya, Tokyo, (1973), 511-520.

[ 4 ] — Q Quasi-coefficient rings of a local ring, Nagoya Math. J., 68 (1977), 123-130.

[5] - Noetherian rings with many derivations, in "Contributions to Algebra" (in honor of E. Kolchin), Academic Press, New York, (1977), 279-294.

[6] Nagata, M., Local rings, Wiley, New York, 1962.

[7] Nomura, M., Formal power series rings over polynomial rings II, in "Number Theory, Algebraic Geometry and Commutative Algebra" (in honor of Y. Akizuki), Kinokuniya, Tokyo, (1973), 521-528.

[ 8 ] Scheja, G. and Storch, U., Differentielle Eigenschaften der Lokalisierungen analytischer Algebren, Math. Ann., 197 (1972), 137-170.

[9] Valabrega, P., On the excellence property for power series rings over polynomial rings, J. Math. of Kyoto Univ., 15 (1975), 387-395.

[E.G.A.] Grothendieck, A. and Dieudonne, J., Eléments de Géométrie Algébrique, Publ. Math. I.H.E.S., $1960 \mathrm{ff}$.

Shotoku-Gakuen Women's Junior College 